\title{
Effect of Metal Ring Setting Outside HTSC Bulk Disk on Trapped Field and Temperature Rise in Pulse Field Magnetizing
}

\author{
Hiroyuki Fujishiro, Kazuya Yokoyama, Masahiko Kaneyama, Tetsuo Oka, and Koshichi Noto
}

\begin{abstract}
In order to enhance the trapped field in cryo-cooled HTSC bulks using pulse field magnetizing (PFM), a metal ring (stainless steel 304 and/or AI) has been tightly set onto the Sm$\mathrm{BaCuO}$ bulk disk and the relation between the total trapped flux $\Phi_{\mathrm{T}}^{\mathrm{P}}$, the trapped field $B_{\mathrm{T}}^{\mathrm{P}}$, and the temperature rise $\Delta T$, has been investigated as a function of the applied pulse field $B_{\text {ex }}$. The $\Phi_{T}^{P}$ and $B_{\mathrm{T}}^{\mathrm{P}}$ values are enhanced about $10 \sim 20 \%$ by the metal ring due to the reduction in the temperature rise $\Delta T$. These results suggest that a part of the generated heat $Q$ due to the flux motion in the peripheral region promptly transfers to the metal ring and the heat transfer to the cold stage is improved by the ring setting.
\end{abstract}

Index Terms-High $T_{\mathrm{c}}$ bulk superconductors, metal ring setting, pinning and viscous loss, pulse field magnetizing, temperature measurement.

\section{INTRODUCTION}

$\mathbf{I}$ $N$ VIEW of the practical applications of high- $T_{c}$ bulk superconductors (HTSCs) as a high strength bulk magnet for a magnetic levitation system and so on, pulse field magnetizing (PFM) as well as the static field-cooled magnetizing (FCM) has been intensively investigated because of the relatively compact and inexpensive setup. The field trapped by PFM is, however, lower than that attained by FCM at temperatures below $77 \mathrm{~K}$. The main reason has been attributed to the large heat generation due to the dynamic motion of the magnetic flux against the vortex pinning force $F_{\mathrm{p}}$ and the viscous force $F_{\mathrm{v}}$. We have studied the temperature rise $\Delta T$ and trapped field $B_{\mathrm{T}}^{\mathrm{P}}$ on the surface of cryo-cooled $\mathrm{YBaCuO}$ and $\mathrm{SmBaCuO}$ bulks during PFM and pointed out the importance of the $\Delta T$ reduction for the $B_{\mathrm{T}}^{\mathrm{P}}$ enhancement [1]-[4]. The total generated heat $Q$ was estimated using the specific heat $C$ of the bulk and the maximum $\Delta T$. It was pointed out that the $B_{T}^{\mathrm{P}}$ values as functions of the initial temperature $T_{\mathrm{s}}$ and applied pulse field $B_{\mathrm{ex}}$ can be understood on the basis of the trapped field $\left(B_{\mathrm{T}}^{\mathrm{FC}}\right)$ by FCM vs.

Manuscript received October 3, 2004. This work was supported in part by Japan Science and Technology Corporation under the Joint-research Project for Regional Intensive in the Iwate Prefecture on Development of practical applications of magnetic field technology for use in the region and in everyday living.

$H$. Fujishiro and M. Kaneyama are with the Faculty of Engineering, Iwate University, Morioka 020-8551, Japan (e-mail: fujishiro@iwate-u.ac.jp; t2203004@iwate-u.ac.jp).

K. Yokoyama is with the National Institute for Materials Science, Tsukuba, 305-0003, Japan (e-mail: Yokoyama.Kazuya@nims.go.jp).

T. Oka is with IMRA Material R\&D Co., LTD., 5-50 Hachiken-cho, Kariya, 448-0021, Japan (e-mail: toka@ai-i.aisin.co.jp).

K. Noto was with the Faculty of Engineering, Iwate University. He is now with Iwate Industrial Promotion Center, Morioka 020-0852, Japan (e-mail: notokoshichi@yahoo.co.jp).

Digital Object Identifier 10.1109/TASC.2005.849425 the temperature $(T)$ diagram, i.e., by the decrease of the critical current density $J_{c}$ associated with the temperature rise. In order to enhance the $B_{\mathrm{T}}^{\mathrm{P}}$ and $\Phi_{\mathrm{T}}^{\mathrm{P}}$ values by PFM, the reduction of the $\Delta T$ is an indispensable issue. The iteratively magnetizing pulsed-field method with reducing amplitude (IMRA) [5], locating yoke pieces around a bulk [6], a use of vortex-type coils [7] and a multi-pulse technique with step-wise cooling (MPSC) [8] have been attempted to suppress the heat generation during PFM. A $B_{\mathrm{T}}^{\mathrm{FC}}$ of $17.24 \mathrm{~T}$ at $29 \mathrm{~K}$ in $\mathrm{YBaCuO}$ bulk has been realized by FCM, enhancing the thermal conduction of the bulk by impregnating a high thermal conductivity alloy into drilled holes [9].

In this study, we set a metal ring ( $\mathrm{Al}$ and/or stainless steel 304) tightly on a SmBaCuO bulk disk. The following effects are expected from the metal ring set on to the HTSC bulk disk. First, since the heat generation due to PFM occurs mainly in the peripheral region, the metal ring attached to the bulk disk periphery can easily and promptly receive the heat. Second, since the $a b$-plane thermal conductivity $\kappa_{\mathrm{ab}}$ is far larger than the $c$-axis $\kappa_{\mathrm{c}}$, the generated heat can easily reach the metal ring along the radial paths of the disk, then transferring to the cold stage through the highly conductive ring. These two effects should contribute to the $\Delta T$ reduction of the bulk. Finally, the metal ring setting helps to mechanically reinforce the bulk disk [10].

\section{EXPERIMENTAL PROCEDURE}

A highly $c$-axis oriented $\mathrm{SmBaCuO}$ bulk superconductor (Dowa Mining Co., Ltd) with $45 \mathrm{~mm}$ diameter and $15 \mathrm{~mm}$ thickness was used. This consisted of four growth sector regions (GSRs), divided by the growth sector boundaries (GSBs) [2]. The bulk is composed of $\mathrm{SmBa}_{2} \mathrm{Cu}_{3} \mathrm{O}_{\mathrm{y}}$ (Sm123) and $\mathrm{Sm}_{2} \mathrm{BaCuO}_{5}(\mathrm{Sm} 211)$ with the molar ratio of Sm123 : Sm211 = $1.0: 0.3,15.0$ wt. $\% \mathrm{Ag}_{2} 0$ powder, and 0.5 wt.\% Pt powder. The bulk was uniformly impregnated by epoxy resin in vacuum and then the epoxy resin layer on the surface of the bulk disk was removed. The stainless steel (SUS304) or the aluminum (Al) ring with $4 \mathrm{~mm}$ in thickness and $15 \mathrm{~mm}$ in height, was fixed onto the bulk disk using apiezon- $\mathrm{N}$ grease. We call the ring-attached sample as SUS-Sm or Al-Sm. Since a large heat generation due to the eddy current took place in the $\mathrm{Al}$ ring $(\Delta T>6 \mathrm{~K}$ at $100 \mathrm{~K})$, the ring was cut with a gap of $1 \mathrm{~mm}$ as shown in Fig. 1. Resultantly, $\Delta T$ was diminished to within $0.2 \mathrm{~K}$. Since the $\mathrm{Al}$ ring did not contact tightly with the bulk due to the gap, another SUS ring 


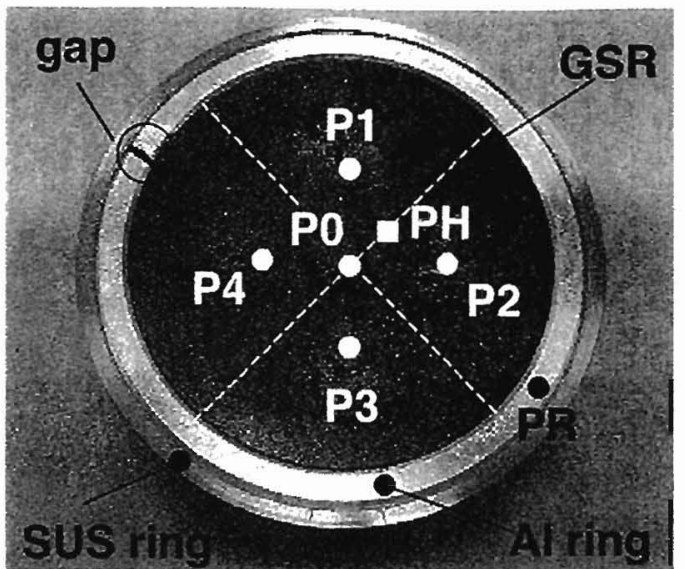

Fig. 1. Photograph of the SmBaCuO-bulk disk fitted with the $\mathrm{W}(\mathrm{Al}+\mathrm{SUS})$-ring. The positions of the temperature and magnetic field measurements $(\mathrm{P} 0 \sim \mathrm{P} 4, \mathrm{PR}$ and $\mathrm{PH})$ are shown.

TABLE I

SUMMARY OF SIZES AND MATERIAL PARAMETERS OF Sm-BULK AND RINGS

\begin{tabular}{lcccc}
\hline \hline & Sm-bulk & SUS-ring & A1-ring & $\begin{array}{c}\text { W-ring } \\
\text { (A1-SUS) }\end{array}$ \\
\hline I.D. $(\mathrm{mm} \phi)$ & - & 45 & 45 & $45-53$ \\
\hline O.D. $(\mathrm{mm} \phi)$ & 45 & 53 & 53 & $53-55$ \\
\hline Height $(\mathrm{mm})$ & 15 & 15 & 15 & $15-15$ \\
\hline$V\left(\mathrm{~mm}^{3}\right)$ & 23.84 & 9.24 & 9.24 & $9.24-2.54$ \\
\hline$C_{\mathrm{r}\left(\mathrm{J} / \mathrm{cm}^{3} \mathrm{~K}\right) \text { at } 40 \mathrm{~K}}$ & 0.48 & 0.38 & 0.23 & - \\
\hline$C(\mathrm{~J} / \mathrm{K})$ at $40 \mathrm{~K}$ & 11.44 & 3.47 & 2.08 & 3.04 \\
\hline$C_{\mathrm{ratio}}$ & 1.0 & 0.30 & 0.18 & 0.27 \\
\hline $\mathrm{K}$ at $40 \mathrm{~K}(\mathrm{~mW} / \mathrm{cmK})$ & $130(\mathrm{ab})$ & 55 & $\sim 5000$ & - \\
\hline \hline & $30(\mathrm{c})$ & & & \\
\hline
\end{tabular}

with $1 \mathrm{~mm}$ in thickness was set outside the Al-ring. Hereafter, we denote this sample with the Al+SUS (W) ring the W-Sm. Table I summarizes the sizes, the volume of each metal ring, and the specific heat $C_{\mathrm{V}}\left(\mathrm{J} / \mathrm{cm}^{3} \mathrm{~K}\right)$, the heat capacity $C(\mathrm{~J} / \mathrm{K})$ at $40 \mathrm{~K}$ of the Sm-bulk and each metal ring, and the ratio of the heat capacity of the metal ring to that of the Sm-bulk. The heat capacity increases by $30 \%, 18 \%$ and $27 \%$ for the SUS-ring, Al-ring and W-ring, respectively, relative to that for the bare Sm bulk. The $a b$-plane and $c$-axis thermal conductivity $\kappa$ at 40 $\mathrm{K}$ of the Sm-bulk and that of SUS and $\mathrm{Al}$ are also shown [11]. $C_{\mathrm{v}}$ and $\kappa$ were measured for the present specimens [12].

The bulk disk was tightly stacked on the sapphire plate ( 45 $\mathrm{mm}$ in diameter and $20 \mathrm{~mm}$ in thickness) attached to the cold stage of a helium refrigerator. The initial stage temperature $T_{\mathrm{s}}$ was kept at $40 \mathrm{~K}$. The temperatures, $T 0$ at the center of the bulk (P0) and, $T 1 \sim T 4$ at $\mathrm{P} 1 \sim \mathrm{P} 4$ were monitored by fine chromel-constantan thermocouples adhered to the upper bulk surface by GE7031 varnish. P1 P4 were situated on the central radial lines of each GSR by $9 \mathrm{~mm}$ apart from P0. The temperature of the metal ring $T R$ was also measured at PR. The total trapped magnetic flux $\Phi_{\mathrm{T}}^{\mathrm{P}}$ and the distribution of the trapped
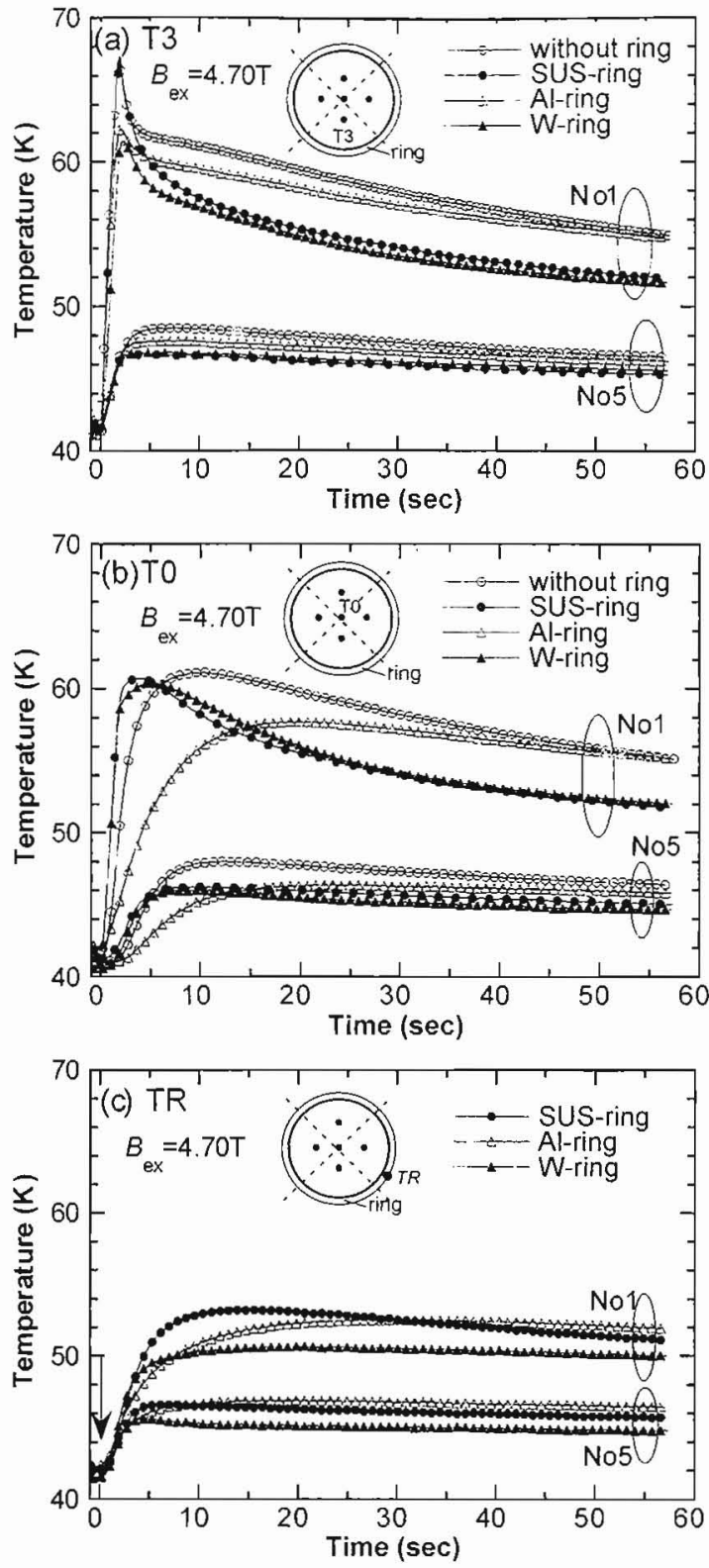

Fig. 2. Examples of the time evolution of temperatures $T(t)$ of the Sm-bulk without ring, with SUS-ring, with Al-ring and with $\mathrm{W}$ (SUS+Al)-ring at (a) T3, (b) $T 0$ and (c) $T R$ after applying the Nol and No5 pulse fields of $B_{\text {ex }}=$ 4.70 T.

magnetic flux density $B_{\mathrm{T}}^{3} \mathrm{~mm}$ were measured using an axial type Hall sensor (F.W. Bell, model BHA 921), which scanned $3 \mathrm{~mm}$ above the bulk surface stepwise with a pitch of $1.2 \mathrm{~mm}$. The trapped field $B_{\Upsilon}^{\mathrm{P}}$ on the bulk surface was measured by the Hall sensor adhered to the position at $\mathrm{PH}$ with a $2.5 \mathrm{~mm}$ distance from P0. Five iterative magnetic pulses (No1 No5) with the same amplitude $B_{\text {ex }}$ from $3.83 \mathrm{~T}$ to $6.04 \mathrm{~T}$ (rise time: $12 \mathrm{~ms}$ ) were applied sequentially after re-cooling to $T_{\mathrm{s}} . T(t), \Phi_{\mathrm{T}}^{\mathrm{P}}$ and $B_{\mathrm{T}}^{\mathrm{P}}$ were measured at each stage.

\section{RESULTS AND DISCUSSION}

Fig. 2(a) shows the time dependence of temperature $T 3(t)$ at P3 after applying the No1 and No5 pulse of $B_{\text {ex }}=4.70 \mathrm{~T}$. For the bare Sm-bulk (B-Sm) without the ring, the magnetic 

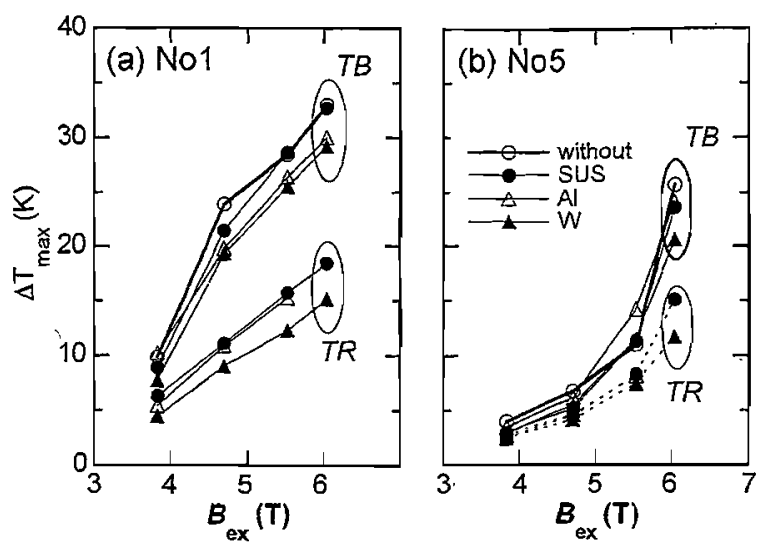

Fig. 3. The maximum temperature rises $\Delta T B_{\max }$ and $\Delta T R_{\max }$ at the bulk and the ring for (a) the No1 and (b) No5 pulses as a function of $B_{\text {ex }}$. The $\Delta T B_{\max }$ values are the averaged ones over $\triangle T 0_{\max }$ to $\Delta T 4_{\max }$.

flux preferentially intrudes into the bulk from the GSRs containing P2, P3 and P4 and the sharp and large temperature rises $(\Delta T=25 \mathrm{~K}$ ) take place within $3 \mathrm{~s}$ at these positions. $T(t) \mathrm{re}$ covers to the initial temperature $T_{s}$ after $15 \sim 20 \mathrm{~min} . T 3(t)$ of SUS-Sm decreases faster after the $T 3(t)$ maximum, although the maximum temperature rise is similar to that of B-Sm. This result means that a part of the generated heat $Q$ promptly transfers to the SUS-ring. The maximum $\Delta T$ value at $T 3$ decreases to $\sim 20 \mathrm{~K}$ for $\mathrm{Al}-\mathrm{Sm}$ and $\mathrm{W}-\mathrm{Sm}$. The rate of temperature decrease $(-d T(t) / d t)$ after the peak is small for Al-Sm but that for the W-Sm is large and almost the same as the SUS-Sm. As for the iterative pulse field applications, $\Delta T$ is the largest for the No1 pulse and decreases for the succeeding pulses [2]. The increment of the trapped field $\Delta B_{\mathrm{T}}^{\mathrm{P}}$ is also the largest for the No1 pulse, followed by a gradual increase for the No2 and No3 pulses. For the No5 pulse application, the $T(t)$ peak disappears for all the settings. $\Delta T$ is slightly smaller for the SUS-Sm and $\mathrm{W}-\mathrm{Sm}$ than for the bare bulk and Al-ring setting.

Fig. 2(b) presents the $T 0(t)$ at $\mathrm{PO}$ for each metal ring setting. $T 0(t)$ rises up latest among $T 0 \sim T 4$ because the distance from the major heat source is usually the longest [2]. For the SUS-Sm and W-Sm, TO( $t)$ reaches a maximum faster than that for B-Sm. These results mean that the flux motion is enhanced in the central region of the bulk by the SUS-Sm and W-Sm and the magnetic fluxes are accumulated faster in the bulk center. The anomalously long time constant for the temperature rise for the Al-ring setting may result from the high thermal contact resistance between the bulk and $\mathrm{Al}$-ring.

Fig. 2(c) shows the $T R(t)$ for each metal ring setting. The maximum $\Delta T$ values for SUS-Sm and W-Sm are $12 \mathrm{~K}$ and $10 \mathrm{~K}$, respectively, and the time constant for the temperature rise of $T R(t)$ is longer for the SUS-Sm and W-Sm than that of $T 3(t)$. The temperature rise in the metal ring is mainly due to the heat conduction from the bulk and the temperature rise in the Al-ring is slower because of the high thermal contact resistance.

Figs. 3(a) and (b) summarize the maximum temperature rise of the bulk $\Delta T B_{\max }$ and the ring $\Delta T R_{\max }$ after the No1 and No5 pulse as a function of $B_{\mathrm{ex}} . \Delta T B_{\mathrm{max}}$, the averaged value at $\Delta T 0_{\max } \sim \Delta T 4_{\max }$, increases with increasing $B_{\mathrm{ex}}$ for both No1 and No5 pulses. $\triangle T B_{\max }$ of $\mathrm{B}-\mathrm{Sm}$ is the largest and is reduced by the metal ring setting. Especially, the reduction of
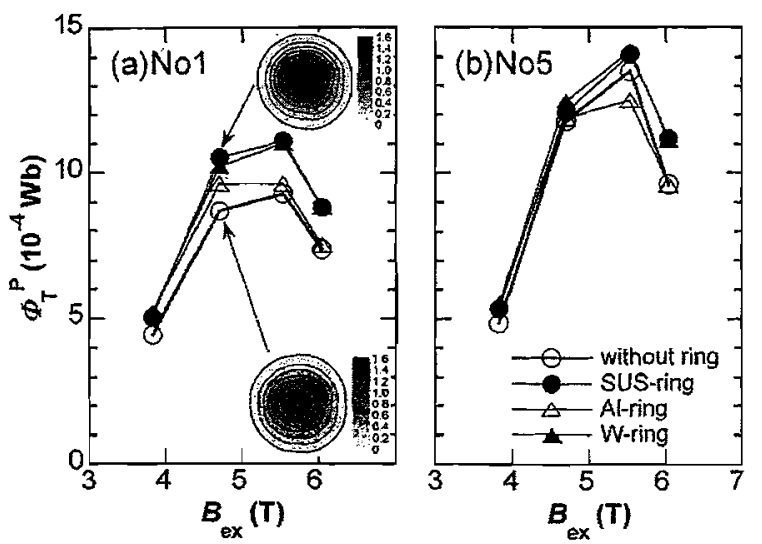

Fig. 4. The total trapped flux $\Phi_{\mathrm{T}}^{\mathrm{P}}$ for each metal ring setting after (a) the Nol and (b) the No5 pulse application, as a function of $B_{\text {ex }}$. The typical conical trapped field distributions $B_{\mathrm{T}}^{3} \mathrm{~mm}$ are displayed for the SUS-ring setting and bare Sm-bulk.

the $\Delta T B_{\max }$ is the largest for W-Sm and Al-Sm; e.g., from $23 \mathrm{~K}$, to $18 \mathrm{~K}$ for the Nol pulse of $B_{\mathrm{ex}}=4.70 \mathrm{~T} . \Delta T R_{\max }$ also increases with increasing $B_{\mathrm{ex}}$ for both Nol and No5 pulses and is about 50 to $80 \%$ of $\triangle T B_{\max } . \Delta T R_{\max }$ is the largest for the SUS-ring and the smallest for the $W$-ring.

Figs. 4(a) and 4(b) present the total trapped flux $\Phi_{\mathrm{T}}^{\mathrm{P}}$ after the No1 and No5 pulse as a function of $B_{\text {ex }}$. For the Nol pulse, $\Phi_{\mathrm{T}}^{\mathrm{P}}$ of B-Sm is smallest for each $B_{\mathrm{ex}}$ and the $\Phi_{\mathrm{T}}^{\mathrm{P}}$ value is enhanced by the metal ring setting. Especially, for the SUS-Sm and W-Sm, the $\Phi_{\mathrm{T}}^{\mathrm{P}}$ values after the $4.70 \mathrm{~T}$ and $5.53 \mathrm{~T}$ pulses increase about $20 \%$ compared with those of the bulk without ring. The increase may mainly come from the decrease of the temperature rise due to the ring setting, which results in the increase of the effective critical current density. The $\Phi_{\mathrm{T}}^{\mathrm{P}}$ enhancement is observed for Al-Sm, which suggests that the good thermal contact between the ring and the $\mathrm{Sm}$-bulk is of vital importance. The trapped field $B_{\mathrm{T}}^{\mathrm{P}}$ measured at $\mathrm{PH}$ is also enhanced by the metal ring setting; for example, $B_{\mathrm{T}}^{\mathrm{P}}(=2.95 \mathrm{~T})$ for $\mathrm{W}-\mathrm{Sm}$ is about $10 \%$ larger than that of B-Sm $(=2.71 \mathrm{~T})$ after the No5 pulse of $B_{\text {ex }}=5.53 \mathrm{~T}$. The typical trapped field distributions $B_{\mathrm{T}}^{3 \mathrm{~mm}}$ are shown in the inset of Fig. 4(a) in which a conical field distribution can be confirmed.

Neglecting the heat drained to the cold stage, the generated heat $Q$ by the PFM operation can be estimated using the following equation,

$$
\begin{aligned}
Q=\int_{T_{s}}^{T_{s}+\Delta T B_{\max }}\left(C_{B u l k} V_{B u l k}\right) d T \\
+\int_{T_{s}}^{T_{s}+\Delta T T_{\max }}\left(C_{R i n g}+V_{R i n g}\right) d T
\end{aligned}
$$

where $C_{\text {Bulk }}$ and $C_{\text {Ring }}$ are the specific heat $\left(\mathrm{J} / \mathrm{cm}^{3} \mathrm{~K}\right)$, and $V_{\text {Bulk }}$ and $V_{\text {Ring }}$ are the volume of the Sm-bulk and the metal ring, respectively. Fig. 5 shows the estimated $Q$ values as a function of $B_{\mathrm{ex}}$ for each metal ring setting. For the Nol pulse, the $Q$ value of the bulk with each metal ring is smaller than that of B-Sm. This result suggests that the heat transferred from the bulk is partially drained to the cold stage through the ring. For 


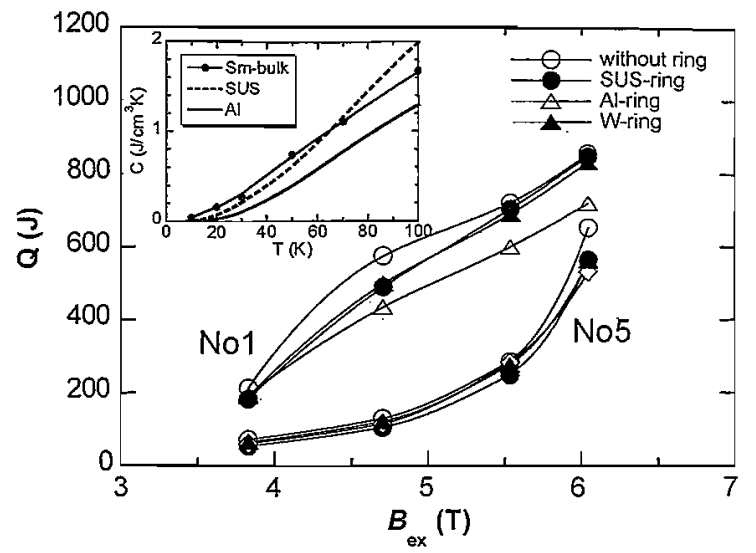

Fig. 5. The estimated generated heat $Q$ as a function of $B_{\mathrm{ex}}$ for each metal ring setting. The inset indicates the heat capacity $C$ of the Sm-bulk, SUS304 and $\mathrm{Al}$.

the No5 pulse, The $Q$ value of B-Sm is nearly the same as the total $Q$ of the bulk and the metal ring, irrespective of the species and volume of the metal ring. This result means that the heat is generated mainly in the bulk and is transferred to the metal ring nearly adiabatically for the No5 pulse. The heat flow to the cold stage may remain small in case $\Delta T B_{\max }$ is not so large.

\section{SUMMARY}

In order to enhance the trapped field in cryo-cooled HTSC bulks by the pulse field magnetizing (PFM) method, the effect of a metal ring set around the $\mathrm{SmBaCuO}$ bulk disk has been investigated by measuring the total trapped flux $\Phi_{\mathrm{T}}^{\mathrm{P}}$, the trapped field $B_{\mathrm{T}}^{\mathrm{P}}$ and the temperature rise $\Delta T$ for the successive application of pulse fields $B_{\text {ex }}$ with various strengths. Important experimental results and conclusions obtained in this study are summarized as follows.

(1) The $\Phi_{\mathrm{T}}^{\mathrm{P}}$ and $B_{\mathrm{T}}^{\mathrm{P}}$ values are enhanced about $10 \sim 20 \%$ by the metal ring setting compared with those of the bare bulk without the metal ring. The main origin is the reduction of $\Delta T$ of the bulk during PFM. Since the heat generation mostly takes place in the peripheral region of the bulk disk in PFM, the metal ring set around the bulk is more effective than the insertion of a high-thermal-conductive alloy on the bulk center.

(2) The SUS- and W(Al+SUS)-rings behave as a good heat reservoir which contribute to the $\Delta T$ reduction in the bulk. The good thermal conductance between the bulk periphery and ring is necessary to reduce $\Delta T$ and to enhance the $\Phi_{\mathrm{T}}^{\mathrm{P}}$ and $B_{\mathrm{T}}^{\mathrm{P}}$ values.

(3) The metal ring with a high thermal conductivity also enhances the thermal drain for the generated heat $Q$ to transfer to the cold stage.

\section{ACKNOWLEDGMENT}

The authors would like to thank Prof. M. Ikebe of Iwate University for the valuable suggestions.

\section{REFERENCES}

[1] H. Fujishiro, T. Oka, K. Yokoyama, and K. Noto, "Time evolution and spatial distribution of temperature in YBCO bulk superconductor after pulse field magnetizing," Supercond. Sci. Technol., vol. 16, pp. 809-814, 2003.

[2] _ "Temperature rise in Sm-based bulk superconductor after applying iterative pulse fields," Supercond. Sci. Technol., vol. 17, pp. 51-57, 2004.

[3] H. Fujishiro, T. Oka, K. Yokoyama, M. Kaneyama, and K. Noto, "Flux motion studies by means of temperature measurement in magnetizing processes for HTSC bulks," IEEE Appl. Supercond., vol. 14, no. 2, pp. 1054-1057, 2004.

[4] H. Fujishiro, K. Yokoyama, M. Kaneyama, T. Oka, and K. Noto, "Estimation of generated heat in pulse field magnetizing for $\mathrm{SmBaCuO}$ bulk superconductor," Physica C, vol. 412-414, pp. 646-650, 2004.

[5] U. Mizutani, T. Oka, Y. Itoh, Y. Yanagi, M. Yoshikawa, and H. Ikuta, "Pulse-field magnetization applied to high- $T_{\mathrm{c}}$ superconductors,". Appl. Supercond., vol. 6, pp. 235-246, 1998.

[6] H. Ikuta, H. Ishihara, Y. Yanagi, Y. Itoh, and U. Mizutani, "Extracting the utmost from the high performance of Sm-Ba-Cu-O bulk superconductors by pulse field magnetization," Supercond. Sci. Tech., vol. 15, pp. 606-612, 2002.

[7] T. Ida, H. Matsuzaki, Y. Akita, M. Izumi, H. Sugimoto, Y. Hondou, Y. Kimura, N. Sakai, S. Nariki, I. Hirabayashi, M. Miki, M. Murakami, and M. Kitano, "Magnetization properties for $\mathrm{Gd}-\mathrm{Ba}-\mathrm{Cu}-\mathrm{O}$ bulk superconductors with a couple of pulsed-field vortex-type coils," Physica $C$, vol. 412-414, pp. 638-645, 2004.

[8] M. Sander, U. Sutter, R. Koch, and M. Kläser, "Pulsed magnetization of HTS bulk parts at $\mathrm{T}<7 \mathrm{i} \mathrm{K}$," Supercond. Sci. Tech., vol. 13, pp. 841-845, 2000.

[9] M. Tomita and M. Murakami, "High-temperature superconductor bulk magnets that can trap magnetic fields of over 17 tesla at $29 \mathrm{~K}$," Nature, vol. 421, pp. 517-520, 2003.

[10] M. Morita et al., "Trapped field and mechanical properties of QMG magnet," in Proc. 1998 Int. Workshop Superconductivity, Tokyo, 1998, pp. 115-118.

[11] H. Fujishiro and S. Kohayashi, "Thermal conductivity, thermal diffusivity and thermoelectric power in Sm-based bulk superconductors," IEEE Trans. Appl. Supercond, vol. 12, pp. 1124-1127, 2002.

[12] M. Ikebe, H. Fujishiro, T. Naito, and K. Noto, "Simultaneous measurement of thermal diffusivity and conductivity applied to Bi-2223 ceramic superconductors," J. Phys. Soc. Jpn., vol. 63, pp. 3107-3114, 1994. 